\title{
Nutzung der Geothermie
}

Gemäß dem 2008 verabschiedeten Klima- und Energiepaket der EU ist Österreich verpflichtet, den Anteil der erneuerbaren Energien am Bruttoenergieverbrauch von derzeit 23\% bis zum Jahre 2020 auf $34 \%$ zu steigern. 2010 wurden von Seiten der Bundesregierung die „Eckpunkte der Energiestrategie Österreich“ veröffentlicht. Demgemäß sollen die ambitionierten Ziele durch die Steigerung der Energieeffizienz, durch Energieeinsparungen und durch den Ausbau der erneuerbaren Energieträger erreicht werden. Obwohl nicht speziell genannt, kann die Nutzung der oberflächennahen Geothermie - besonders bei Umsetzung moderner Raumwärmekonzepte mit Niedertemperaturheizung und Hochtemperaturkühlung - sehr wesentlich zur Zielerreichung im Bereich Wärme beitragen. Die Studie GEO-Pot (Präsentation der Ergebnisse am 16. April 2010) beziffert das technische Anwendungspotenzial der Geothermie mit mind. 66 PJ/a und somit deutlich über dem Deckungspotenzial von 55 PJ/a. Demgemäß liegt das technische Anwendungspotenzial der Geothermie wesentlich über dem forstlichen Biomassepotenzial von 50 PJ (Quelle: Eckpunkte der Energiestrategie Österreich, 2010).

Die Nutzung der Erdwärme hat gerade im letzten Jahrzehnt einen großen Aufschwung erlebt. Gründe dafür sind das hohe technische Niveau der Anlagen, verbesserte Kenntnisse der thermischen Eigenschaften des Untergrunds sowie die flächendeckende Verfügbarkeit für wirtschaftliches Heizen und Kühlen energieeffizienter Gebäude. Deshalb wurden auch von Seiten des ÖWAV die bestehenden Regelwerke überarbeitet und 2009 im ÖWAV-Regelblatt 207 „Thermische Nutzung des Grundwassers und des Untergrundes - Heizen und Kühlen“ neu herausgegeben.

In diesem Regelblatt werden sowohl für offene Systeme (d. h. Entnahme und Rückgabe von Grundwasser) als auch für geschlossene Systeme (Erdwärmekollektoren an der Oberfläche, Erdwärmesonden mit einer Tiefe von zumeist 70 bis $150 \mathrm{~m}$ et al.) Hilfestellungen für Planung und Betrieb gegeben. Dies beinhaltet auch einfache Berechnungswerkzeuge, die stark vereinfachte Berechnungsannahmen voraussetzen. Die bisherige Praxis zeigt, dass diese Berechnungswerkzeuge breite Anwendung finden, fallweise aber auch dort eingesetzt werden, wo dem Planer die Verwendung numerischer Planungswerkzeuge anzuraten ist.

Das gegenständliche Heft der Österreichischen Wasser- und Abfallwirtschaft soll neue Erkenntnisse aus Forschung und Praxis der „Nutzung der Geothermie“ einer interessierten Fachöffentlichkeit vorstellen.

- Im ersten Artikel geben Adam und Markiewicz einen umfassenden Überblick über das Prinzip der Nutzung der Geothermie, zeigen Berechnungswerkzeuge, aber auch verschiedene neue Anwendungen von Absorbersystemen in Bauwerken.

- Die nächsten beiden Artikel beschäftigen sich mit Erdwärmesonden. Aschauer stellt in seinem Beitrag die Auswertung der Betriebsdaten von Erdwärmesonden der Heizperiode 2008/09 und den davon abgeleiteten Wärmeleitfähigkeitskataster von Oberösterreich vor. Niederbrucker stellt

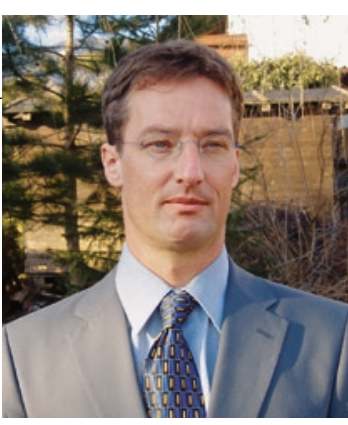

Univ.-Prof.

DI Dr. Wolfgang Rauch

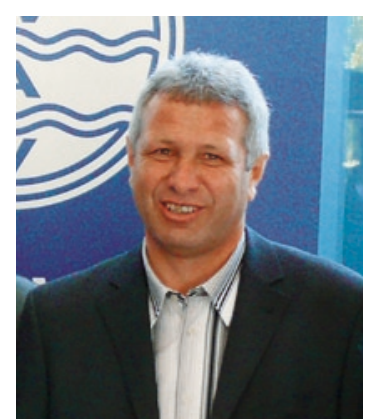

DI Johann Aschauer 
direkte und indirekte Messmethoden für die Bestimmung der für die Bemessung von Erdwärmesonden maßgeblichen Bodenkennwerte Wärmeleitfähigkeit und Wärmekapazität vor und analysiert im zweiten Teil der Arbeit die Eigenschaften von Verpressmaterialien.

- Huber et al. zeigen in Ihrem Artikel ein innovatives Monitoringkonzept von Direktverdampferanlagen und dokumentieren hohe Jahresarbeitszahlen.

- Die letzten beiden Arbeiten beschäftigen sich mit dem Potenzial der Erdwärmenutzung. Urich et al. analysieren hierbei den Einfluss der Siedlungsstruktur auf das real erzielbare Potenzial von Grundwasserwärmenutzungen (offene Systeme) und berechnen das Potenzial mit ca. $15 \%$ des Wärmebedarfs. Götzl et al. stellen die Evaluierung des oberflächennahen geothermischen Nutzungspotenzials von geschlossenen Systemen auf Grundlage eines Rasterzellenansatzes dar. Die Quantifizierung des Potenzials war noch nicht abgeschlossen.

Wie die gegenständlichen Beiträge zeigen, kann die Geothermie einen wesentlichen Beitrag zur Erreichung des Zieles von $34 \%$ Erneuerbare Energien bis 2020 leisten. Die Arbeiten stellen das hohe Niveau und die Möglichkeiten der Nutzung der Geothermie dar, identifizieren aber auch noch bestehende Defizite und zukünftige Aufgabenstellungen.

Univ.-Prof. DI Dr. Wolfgang Rauch

Universität Innsbruck

Arbeitsbereich Umwelttechnik

Technikerstraße 13

6020 Innsbruck

E-Mail: wolfgang.rauch@uibk.ac.at

\section{Johann Aschauer}

Amt der Oberösterreichischen Landesregierung

Direktion Umwelt und Wasserwirtschaft

Abteilung Grund- und Trinkwasserwirtschaft

Kärntnerstraße 10-12

4021 Linz

E-Mail: johann.aschauer@ooe.gv.at 